\title{
Defining the Location of T-bet-expressing Myeloid Cells During Acute Intestinal Toxoplasma gondii Infection
}

\author{
Melody Wickstrom ${ }^{1}$, Madison Schanz², Kimberly Larson ${ }^{1,2}$, Américo López-Yglesias $^{3}$ \\ ${ }^{1}$ Indiana University School of Medicine; ${ }^{2}$ Indiana State University; ${ }^{3}$ Indiana University School of \\ Medicine, Department of Microbiology and Immunology
}

\section{Background/Objective:}

The protozoan parasite Toxoplasma gondii is the second leading cause of foodborne pathogenrelated deaths in the United States. The transcription factor T-bet is indispensable for host immunity against $\mathrm{T}$. gondii. The absence of $\mathrm{T}$-bet results in rapid susceptibility during parasite infection. T-bet has been considered essential for T-cell-derived IFN- $\gamma$ during T. gondii infection; yet, recent research has shown that T-bet is not required for lymphocyte-derived IFN- $\gamma$ responses. Our preliminary research shows that $\mathrm{T}$-bet-deficient mice succumb to parasite infection significantly quicker than mice lacking lymphocytes. This has led to our hypothesis that T-bet-dependent myeloid cells are critical for host resistance during acute intestinal T. gondii infection. The objective of this project was to define the location of the T-bet-expressing myeloid cells in the medial small intestines (MSI) of naïve and infected mice during acute mucosal parasite infection.

\section{Methods:}

We used immunofluorescence microscopy to determine the location of T-bet-expressing myeloid cells in the MSI of naïve and T. gondii infected mice. Mice were orally infected with 40 cysts of the ME49 strain of $T$. gondii. On days 0 and 5, one-inch MSI segments were harvested, fixed with $4 \%$ paraformaldehyde for at least one hour, and then frozen in OCT compound. Tissues were then cut into $8 \mu \mathrm{m}$ sections and placed onto slides for staining. Sections were stained for nuclei, CD11c, T-bet, and T. gondii.

\section{Results:}

Our results revealed T-bet-expressing CD11c+ cells in both the MSI and spleen on days 0 and 5 of $T$. gondii infection.

\section{Summary:}

These data indicate that T-bet-expressing myeloid cells are present in the MSI during T. gondii infection. Defining the position of these cells will allow us to determine T-bet's role in mediating myeloid cell-dependent $T$. gondii clearance. Due to the limited treatment options for patients suffering from toxoplasmosis it is critical to define new mechanisms for eliminating T. gondii. 